\title{
Relación biogeográfica entre cangrejos dulceacuícolas y acociles a lo largo de la zona mexicana de transición: revaluación de la hipótesis de Rodríguez (1986)
}

\section{Biogeographic relationship of freshwater crabs and crayfish along the Mexican transition zone: reevaluating Rodríguez (1986) hypothesis}

\author{
Fernando Álvarez ${ }^{凶}$, José Luis Villalobos, Gema Armendáriz y Carmen Hernández \\ Colección Nacional de Crustáceos, Instituto de Biología, Universidad Nacional Autónoma de México, Apartado postal 70-153, 04510 México D. F., \\ México. \\ $\triangle$ falvarez@unam.mx
}

\begin{abstract}
Resumen. A partir de la hipótesis planteada por Rodríguez (1986) sobre una posible exclusión biogeográfica entre acociles de la familia Cambaridae y cangrejos de agua dulce de la familia Pseudothelphusidae a lo largo del centro y sur de México, se han hecho varios estudios que toman en cuenta este patrón. Sin embargo, no se han examinado los patrones de distribución de estos grupos ni cuáles son las zonas ni a qué grado se da el traslape o contacto de las 2 distribuciones. Se construyeron 2 bases de datos, una con más de 1400 registros de las especies de la familia Cambaridae y otra con más de 400 registros de cangrejos de la familia Pseudothelphusidae. Se construyeron mapas de distribución y se identificaron 3 regiones donde el contacto es evidente: el eje neovolcánico, la región de Los Tuxtlas, Veracruz y el estado de Chiapas. Los resultados muestran grandes zonas de contacto en las cuales tanto acociles como cangrejos pueden estar en la misma localidad y en el mismo cuerpo de agua o pueden estar en 2 localidades muy cercanas pero con una diferencia altitudinal. En general, los acociles tienden a distribuirse a mayores altitudes que los cangrejos. Aunado a los patrones de distribución, se discute que aunque los 2 grupos estén presentes en la misma localidad y en el mismo cuerpo de agua, el microhábitat que ocupa cada uno es distinto, por lo que se sugiere que la interferencia entre ellos será poca o nula.
\end{abstract}

Palabras clave: acocil, Cambaridae, cangrejo de agua dulce, Pseudothelphusidae, exclusión biogeográfica.

\begin{abstract}
After Rodríguez (1986) hypothesis on the possible biogeographic exclusion between crayfish of the family Cambaridae and freshwater crabs of the family Pseudothelphusidae throughout central and southern Mexico, several studies have considered it as a real pattern. However, the distribution patterns of both groups have not been examined, nor in what areas and to what extent the overlap of the 2 distributions occurs. Two data bases were compiled, one with more than 1400 records of the species of the family Cambaridae and a second one with more than 400 records of freshwater crabs of the family Pseudothelphusidae. The resulting distribution maps show 3 areas where the contact is evident: the neovolcanic axis, Los Tuxtlas region, Veracruz, and the state of Chiapas. The results show large areas of contact in which both, crayfishes and crabs, can occur in the same locality and in the same body of water, or cases in which they occur in 2 nearby localities with an altitudinal difference. In general crayfishes tend to be distributed at higher altitudes than crabs. Together with the distribution patterns we discuss that the particular microhabitat of each group within the body of water prevents any interference or direct competition.
\end{abstract}

Key words: crayfish, Cambaridae, freshwater crab, Pseudothelphusidae, biogeographic exclusion.

\section{Introducción}

Los cangrejos de agua dulce de la familia Pseudothelphusidae con 60 especies (Villalobos y Álvarez, 2008; Álvarez et al., 2011) y los acociles de la familia Cambaridae con 56 especies (Armendáriz, 2011) son elementos conspicuos de la fauna dulceacuícola mexicana. Los cangrejos pseudotelfúsidos se distribuyen en México

Recibido: 09 noviembre 2011; aceptado: 19 abril 2012 siguiendo un patrón estrictamente neotropical. Se distribuyen desde el norte del Perú y el centro de Brasil hacia el norte hasta México, incluyendo las Antillas, excepto Jamaica (Rodríguez, 1982). En particular en México su distribución alcanza, por la vertiente del Pacífico, los estados de Sonora y Sinaloa; a través del centro del país se encuentran en Nayarit, Durango, Jalisco, Michoacán, Guanajuato, Hidalgo y Puebla; sobre la vertiente del golfo de México se encuentran hasta la porción central de Veracruz (Villalobos y Álvarez, 2008). Los pseudotelfú- 
sidos que ocupan el resto del sur de México se encuentran en áreas generalmente por debajo de los $1000 \mathrm{~m}$ de altitud y no penetran a la península de Yucatán (Fig. 1A) (Huidobro et al., 2006). Se ha discutido que la falta de cuerpos de agua superficiales en la península ha limitado su expansión en esa región (Rodríguez, 1982, 1986); sin embargo, otros grupos estrictamente dulceacuícolas si lo han hecho (Botello y Álvarez, 2010), sugiriendo que puede haber otros procesos relacionados con la biogeografía histórica que pueden explicar este patrón.

En contraste, los acociles de la familia Cambaridae se distribuyen fundamentalmente en Norteamérica, desde el sur de Canadá, ocupando principalmente la porción este de los Estados Unidos de América (EUA), pero con presencia sobre la costa oeste (Hobbs, 1989). Se ha propuesto como un importante centro de radiación de los cambáridos, la región sureste de los EUA, donde se presenta una alta concentración de especies (Hobbs, 2001). En México, los cambáridos se encuentran en la vertiente del golfo de México, a lo largo del eje neovolcánico transversal, algunas especies en el centro y norte del país, en Chiapas y la península de Yucatán (Fig. 1B). Es interesante notar que la distribución de acociles en Chiapas aparentemente ocurre en las porciones de las cuencas que conectan al golfo de México y no ha podido invadir las cuencas que vierten hacia el Pacífico. Al sur de México, 2 especies alcanzan Guatemala, una Belice y otra más Honduras; en el Caribe 4 especies se distribuyen en Cuba (Villalobos, 1982; Hobbs, 1989).
Los patrones de distribución de las 2 familias claramente entran en contacto en varias zonas: a lo largo del centro de México sobre el eje neovolcánico transversal, en la región de Los Tuxtlas al sur de Veracruz (Álvarez et al., en prensa), en la zona serrana de Tabasco (Álvarez et al., 2005) y en varias localidades en diferentes provincias fisiográficas de Chiapas. Este sobrelapamiento parcial de las áreas de distribución y una aparente exclusión espacial de los 2 grupos llevó a Rodríguez (1986) a proponer la exclusión biogeográfica entre los cangrejos de la familia Pseudothelphusidae y los acociles de la familia Cambaridae.

De manera específica, Rodríguez (1986) argumentó que la zona de contacto de las distribuciones de cangrejos y acociles se presenta a lo largo de un frente de $3000 \mathrm{~km}$ en México y Guatemala. Expuso que aunque el traslape de las distribuciones solamente ocurría en 2 áreas, el lago de Chapala y la planicie costera de Veracruz, esto sugería que un grupo había excluido al otro y se aceptaba que los pseudotelfúsidos habían desplazado a los cambáridos que en el pasado habrían tenido un área de distribución mayor. Además de una figura que representaba las áreas de distribución de ambos grupos en México y Guatemala, Rodríguez (1986) se apoyó en una publicación de Holt (1964) en la que se propone el hipotético hallazgo en ese momento de un branquiobdélido (Annelida) parásito en el cangrejo pseudotelfúsido Ptychophallus tumimanus (Rathbun, 1898), que hasta entonces sólo se conocía de acociles. Para explicar la nueva presencia de branquiob-

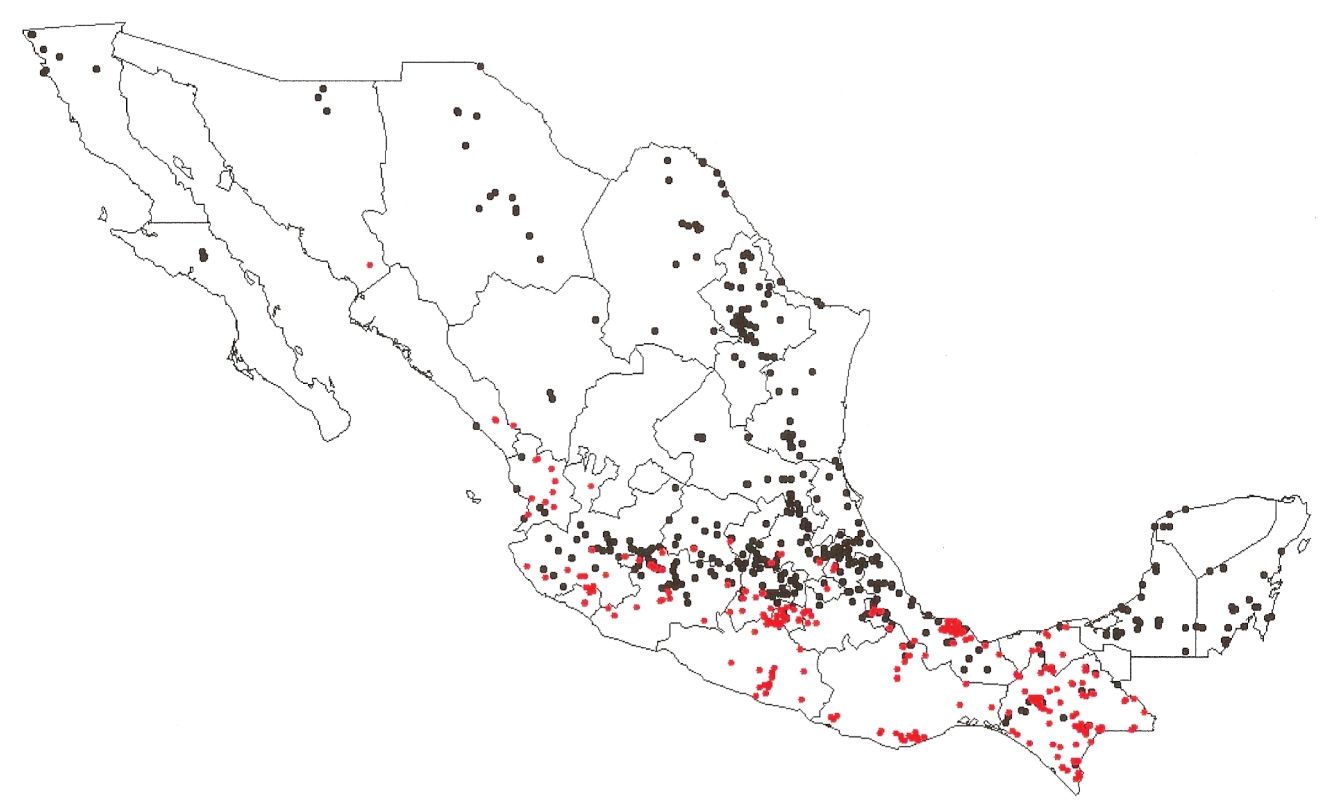

Figura 1. Distribución de los cangrejos de agua dulce de la familia Pseudothelphusidae y de los acociles de la familia Cambaridae en México. Círculos negros, acociles; círculos naranja, cangrejos. 
délidos en pseudotelfúsidos, Holt (1964) propuso que éstos habrían sido transmitidos de acociles a cangrejos en Costa Rica y que después los cangrejos habrían restringido el área de distribución de los acociles, por medio de competencia directa, hasta Guatemala y Honduras.

La hipótesis de Holt (1964) no está sustentada en evidencia concreta y hasta ahora no se ha registrado que haya habido especies de acociles nativos en Costa Rica. Por otra parte, hoy se tienen registros de branquiobdélidos de otros cangrejos pseudotelfúsidos en Tehuana veracruzana (Rodríguez y Smalley, 1969) del río Tapalapa, Santiago Tuxtla, Veracruz, México y en Potamocarcinus nicaraguensis Rathbun, 1893, de la isleta de Granada, lago de Nicaragua, Nicaragua (Gelder, 1999). Estos hallazgos implican que los branquiobdélidos podrían tener una distribución continua desde Canadá hasta Costa Rica y no es necesario invocar mecanismos como el propuesto por Holt (1964) para explicar su presencia en Centroamérica.

Es interesante que Pretzmann (1987), al igual que Rodríguez (1986), formulara para la cuenca del Mediterráneo la hipótesis de que la presente distribución geográfica del cangrejo potámido Potamon fluviatile (Herbst, 1785) y del astácido Austropotamobius pallipes (Lereboullet, 1858) es el resultado de una exclusión competitiva en la cual el cangrejo ha forzado al acocil a contraer su área de distribución. Pretzmann (1987) consideró que las distribuciones de las 2 especies han entrado en contacto varias veces desde el Pleistoceno y que los cangrejos han persistido en áreas con temperaturas más altas.

Por otra parte, aunque existe una vasta literatura sobre competencia interespecífica en acociles, sobre todo entre especies introducidas y especies nativas (Hill y Lodge, 1999; Nakata y Goshima, 2003; Gherardi y Daniels, 2004), no se tienen antecedentes respecto a la competencia directa entre cangrejos pseudotelfúsidos y acociles de la familia Cambaridae. Solamente se pueden citar un par de estudios, que se originan en las ideas de Pretzmann (1987), sobre la competencia entre el cangrejo Potamon fluviatile y el astácido Austropotamobius pallipes (Barbaresi y Gherardi, 1997); y entre estos mismos, con la adición del cambárido Procambarus (Scapulicambarus) clarkii Girard, 1852 (Gherardi y Cioni, 2004). Los resultados de ambos estudios indican que el cangrejo $P$. fluviatile fue superior a $A$. pallipes y $P$. (S.) clarkii al competir por refugio y comida. Los 2 estudios se llevaron a cabo en condiciones de laboratorio. Son necesarias observaciones de campo que pudieran corroborar la existencia de esta interacción en la naturaleza.

En el caso de la interacción entre cangrejos pseudotelfúsidos y acociles cambáridos también hay que considerar cuál es el microhábitat que cada uno ocupa en un cuerpo de agua y cuáles las diferencias en comportamiento y fisiológicas para evaluar si es posible que exista competencia entre ambos. En México, los cangrejos pseudotelfúsidos se encuentran preferentemente en las orillas de los cuerpos de agua bajo rocas, bajo la hojarasca o vegetación presente. Los pseudotelfúsidos, al igual que otros grupos de cangrejos de agua dulce de África y Australia, poseen un epitelio especializado y altamente vascularizado llamado pseudo-pulmón, que recubre la cámara branquial y les permite la respiración aérea mientras ésta se conserve húmeda (Díaz y Rodríguez, 1977; Taylor y Greenaway, 1979; Cumberlidge, 1991). Utilizando las ventajas que les provee esta estructura, los pseudotelfúsidos son capaces de vivir desde las orillas de un cuerpo de agua y zonas adyacentes, hasta en zonas húmedas del suelo de bosques y selvas a cientos de metros de algún cuerpo de agua (Álvarez, 1989).

Por otra parte, aunque los cambáridos son organismos que en ocasiones pueden salir, habitualmente se encuentran en el fondo del cuerpo de agua, en la capa superficial de sedimento o asociados a las raíces de la vegetación riparia; muchas especies cavan galerías y viven dentro por periodos variables. Hobbs (1981) propuso clasificar los acociles por sus hábitos enterradores, dependiendo del tiempo que pasan en la madriguera; serían primarios los que salen de la madriguera raramente; secundarios los que salen por temporadas largas, como la de lluvias, y terciarios aquellos que viven en aguas abiertas pero se esconden en madrigueras. En los enterradores primarios, la complejidad y el largo de las galerías de las madrigueras es mayor; en los terciarios son más simples y cortas. Cada tipo de hábito enterrador se presenta en un tipo determinado de terreno: los enterradores primarios se encuentran en áreas pantanosas donde el nivel freático no tiene variaciones considerables a lo largo del año; los secundarios se presentan en zanjas o depresiones que se inundan estacionalmente y los terciarios se encuentran en cuerpos de agua abiertos.

La clasificación de Hobbs (1981) y una creciente literatura sobre la bio-perturbación que ocasionan las madrigueras muestran la relevancia de este hábito enterrador en los acociles de la familia Cambaridae y su importancia en la hidrología de ciertas regiones (Correia y Ferreira, 1995; Butler, 2002; Holdich y Black, 2007). En contraste, hasta ahora no se ha registrado que los cangrejos pseudotelfúsidos excaven madrigueras; más bien, para ocultarse, buscan oquedades en las orillas de los ríos $\mathrm{y}$ entre las rocas que dejan espacios húmedos.

Con el propósito de explorar la hipótesis de Rodríguez (1986) se podrían seguir por lo menos 2 aproximaciones. La primera consistiría en construir una distribución para todas las especies de acociles y otra para todas las espe- 
cies de cangrejos, de manera que se pudieran identificar las áreas de contacto o traslape entre los 2 grupos. Este tipo de evidencia podría indicar, dependiendo de la extensión del área de traslape, si ha habido una interacción entre los 2 grupos y si de haber existido una exclusión competitiva, uno ha reducido o modificado el área de distribución del otro. Una segunda posibilidad sería la de efectuar observaciones de campo y/o experimentos en hábitats controlados, sobre la competencia directa entre cangrejos y acociles, que pudiera apoyar un escenario de exclusión competitiva.

En este estudio, haciendo uso de una base de datos que contiene la mayoría de los registros publicados de cangrejos y acociles de México y de registros de colecciones biológicas, se examinan las zonas de contacto entre las distribuciones de los 2 grupos. El planteamiento, además de considerar la identificación de áreas de contacto o traslape de los 2 grupos, incluye la compilación de una lista de localidades donde, hasta ahora, se hayan encontrado tanto cangrejos como acociles y un análisis altitudinal de la distribución de ambos grupos en algunas zonas.

Cabe añadir un comentario más acerca de la relevancia de analizar esta hipótesis. La resistencia biótica y la competencia directa por recursos pueden verse influidas de manera distinta si cangrejos y acociles coexisten o si se excluyen. En cualquiera de los 2 escenarios es interesante conocer cuál es el patrón de distribución de los grupos y si hay interacción entre ellos, para después poder asociarlo con la presencia de especies exóticas que serían competidores directos (e.g., acociles cambáridos como Orconectes (Gremicambarus) virilis Hagen, 1870 y Procambarus (S.) clarkii, o el parastácido Cherax quadricarinatus Von Martens, 1868, o especies invasivas que podrían ser presas de ambos grupos (e.g., gasterópodos Tarebia granifera (Lamarck, 1822) como presas). Las comunidades pueden tener una mayor resistencia biótica si los procesos que se dan son más diversos y si no se ha empobrecido perdiendo especies debido a factores externos (Levine et al., 2004). Más aún, se ha propuesto que una combinación de depredadores en el medio dulceacuícola podría ser una barrera eficiente contra especies invasoras (Harvey et al., 2004), idea que de tener cangrejos y acociles coexistiendo en el mismo cuerpo de agua podría aplicarse directamente.

Por otra parte, en estudios de grandes patrones de distribución y especiación en el medio dulceacuícola se ha mencionado la importancia de un conocimiento más profundo sobre la interacción que puede haber entre distintos grupos de depredadores que coexisten. En un estudio reciente (Covich, 2010) se discute si la interacción entre acociles, cangrejos y langostino, considerando los 3 grupos conjuntamente, ha afectado la evolución de los caracoles de agua dulce. De esta manera, se hace evidente la importancia que revisiones como la presente puede tener.

\section{Materiales y métodos}

Se construyó una base de datos que contiene todas las especies de acociles y cangrejos pseudotelfúsidos que se distribuyen en México. Para cada especie se consideraron las localidades georreferenciadas y su altitud. En especies con intervalos amplios de distribución en áreas que no son de interés; por ejemplo, de acociles en Tamaulipas, Nuevo León y Coahuila, donde no hay cangrejos de agua dulce, sólo se consideraron las localidades que aportaban información nueva. Esto es, algunas localidades muy cercanas unas de otras no fueron incluidas en la base de datos ni en el análisis altitudinal.

La información se deriva principalmente de los registros contenidos en la Colección Nacional de Crustáceos (CNCR) del Instituto de Biología, Universidad Nacional Autónoma de México y en la Colección de Crustáceos del Smithsonian Institution (USNM), Washington, D.C. Otras fuentes importantes de datos fueron: Campos y Rodríguez-Almaraz (1992), Villalobos (2005), Villalobos y Álvarez (2008), Rodríguez-Almaraz y Muñiz-Martínez (2008) y Álvarez et al. (2011). La base de datos de acociles contiene 1419 registros de 56 especies y la de cangrejos 508 registros de 60 especies. Todos los registros fueron revisados cartográficamente y/o mediante el programa Google Earth para asegurar que son congruentes con la distribución conocida o posible de la especie y en caso de duda se verificó la identificación de los organismos.

Una vez que se tuvieron las bases de datos completas se construyó un mapa de distribución para ambos grupos (Fig. 1). A partir de éste se seleccionaron 3 áreas geográficas para explorar con mayor detalle el contacto o traslape de las distribuciones que son: el eje neovolcánico transversal, la región de Los Tuxtlas, Veracruz y el estado de Chiapas (Figs. 2,3). Adicionalmente se obtuvieron las altitudes de todas las localidades incluidas en estas 3 regiones para determinar si existe una segregación por altitud entre acociles y cangrejos.

Se buscaron aquellas localidades donde se registraron tanto cangrejos como acociles y se revisaron los datos de recolección específicos para determinar si ambos grupos procedían exactamente del mismo sitio, entendiendo esto como del mismo cuerpo de agua. Los casos en que se determinó que la coincidencia existía se listan en el Cuadro 1.

\section{Resultados}

Los mapas muestran las distribuciones completas de acociles y cangrejos. Los acociles se encuentran en la 


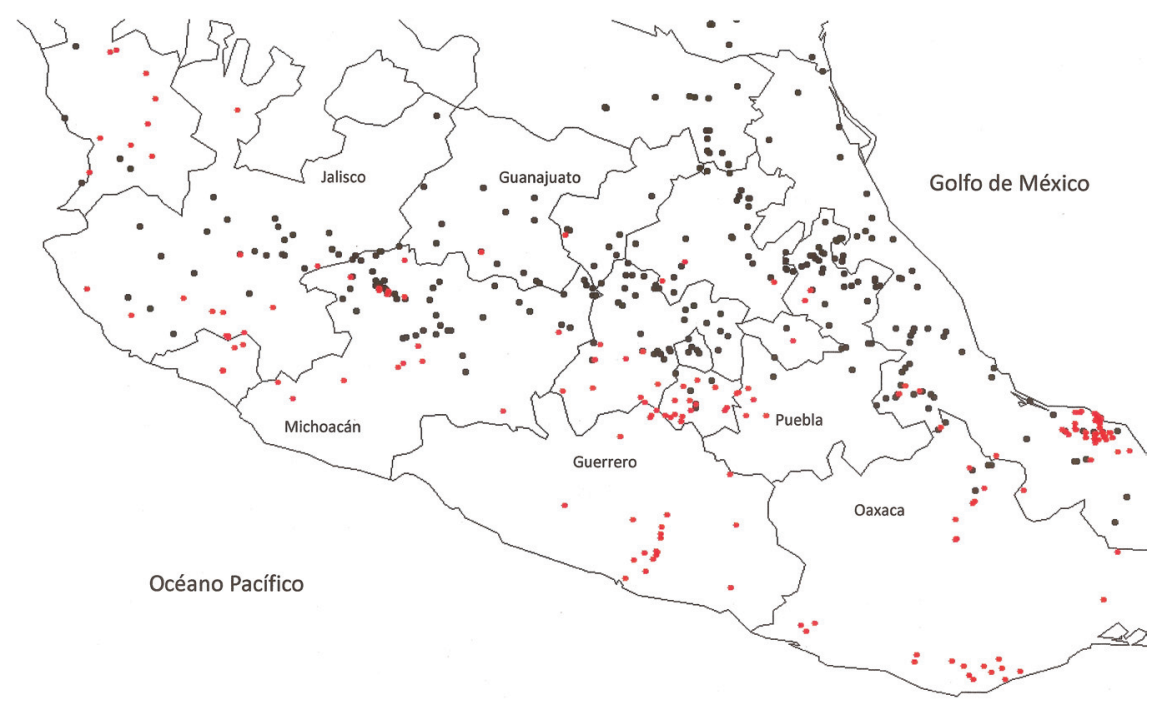

Figura 2. Distribución de los cangrejos de agua dulce de la familia Pseudothelphusidae (círculos naranja) y de los acociles de la familia Cambaridae (círculos negros) en el centro de México.

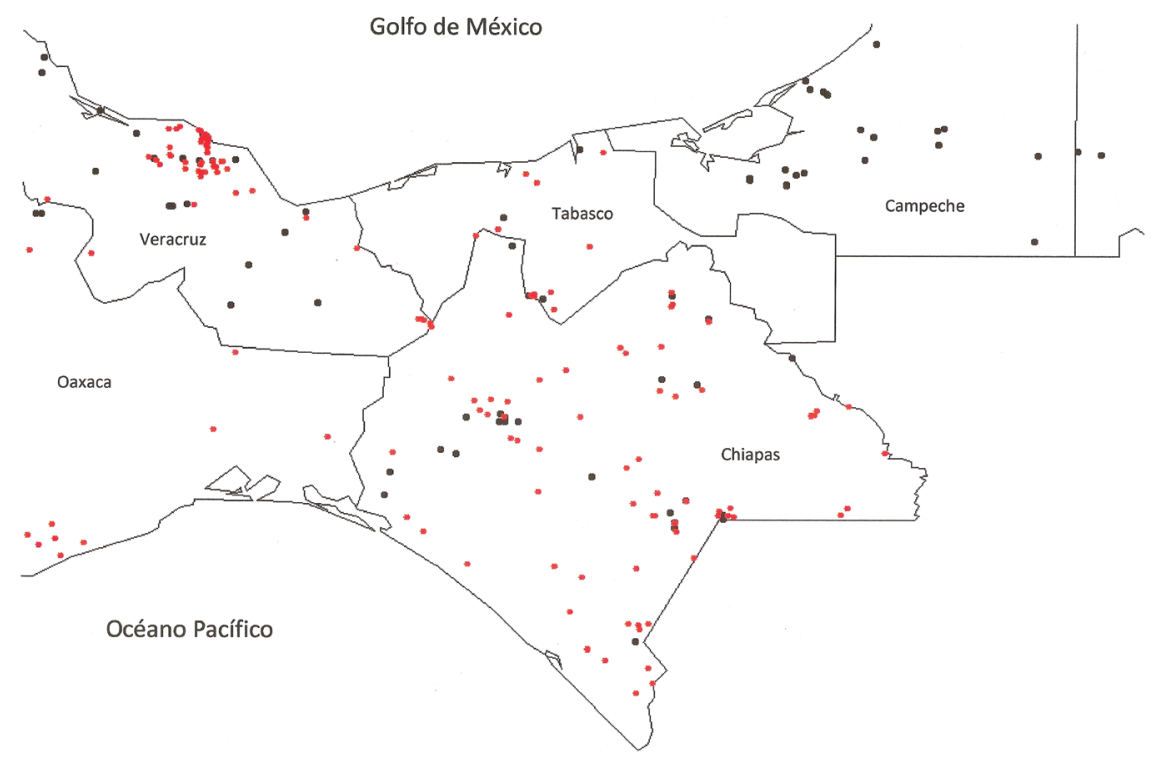

Figura 3. Distribución de los cangrejos de agua dulce de la familia Pseudothelphusidae (círculos naranja) y de los acociles de la familia Cambaridae (círculos negros) en el sur de México.

mayoría de los estados del país; están ausentes de Aguascalientes, Guerrero y Zacatecas (Fig. 1). Es muy probable que la presencia de acociles, debido al acocil rojo Procambarus (S.) clarkii, en Durango, Chihuahua, Baja California y Baja California Sur, se deba a introducciones recientes que no representan localidades dentro de la distribución original de la especie (Campos y Rodríguez-Almaraz, 1992; Torres y Álvarez, 2012).
Los cangrejos tienen un patrón de distribución estrictamente neotropical y hasta donde se sabe no ha habido introducciones que pudieran expandir el área de distribución del grupo. Los cangrejos pseudotelfúsidos están ausentes de la península de Baja California, de Chihuahua, Coahuila, Nuevo León, Tamaulipas, Zacatecas, Aguascalientes, San Luis Potosí y de la península de Yucatán (Fig. 1). 
Cuadro 1. Localidades donde se han recolectado cangrejos de agua dulce de la familia Pseudothelphusidae y acociles de la familia Cambaridae

\begin{tabular}{|c|c|c|c|c|c|}
\hline & Localidad & Estado & Cangrejo & Acocil & Referencia \\
\hline 1 & Villa de las Rosas & Chiapas & Raddaus sp. & Procambarus llamasi & CNCR \\
\hline 2 & San Rafael del Arco & Chiapas & $\begin{array}{l}\text { Raddaus bocourti, Raddaus sp., } \\
\text { Phrygiopilus montebelloensis }\end{array}$ & Procambarus mirandai & CNCR \\
\hline 3 & Bochil & Chiapas & Raddaus bocourti & Procambarus sbordonii & CNCR \\
\hline 4 & Rayón & Chiapas & Raddaus bocourti & Procambarus sp & CNCR \\
\hline 5 & Ruinas de Toniná & Chiapas & Odontothelphusa toninae & Procambarus sp. & $\begin{array}{l}\text { CNCR, Álvarez } \\
\text { y Villalobos } \\
\text { (1991) }\end{array}$ \\
\hline 6 & Berriozábal & Chiapas & Raddaus sp. & Procambarus sp. & CNCR \\
\hline 7 & Montebello & Chiapas & Raddaus bocourti & Procambarus mirandai & CNCR \\
\hline 8 & Río Mezcalapa & Chiapas & Potamocarcinus hartmanni & Procambarus sp. & CNCR \\
\hline 9 & Mixquiahuala & Hidalgo & Pseudothelphusa americana & Cambarellus montezumae & CNCR \\
\hline 10 & Río Santiago, Amatitán & Jalisco & Pseudothelphusa terrestris & Cambarellus montezumae & CNCR \\
\hline 11 & Lago de Chapala & Jalisco & Pseudothelphusa jouyi & $\begin{array}{c}\text { Cambarellus chapalanus, } \\
\text { C. prolixus, Procambarus } \\
\text { digueti }\end{array}$ & CNCR \\
\hline 12 & Cuautitlán & Jalisco & Pseudothelphusa seiferti & Cambarellus montezumae & CNCR \\
\hline 13 & El Grullo & Jalisco & Pseudothelphusa sp. & Cambarellus montezumae & CNCR \\
\hline 14 & Valle de Bravo & México & Pseudothelphusa mexicana & Cambarellus montezumae & CNCR \\
\hline 15 & Chilchota & Michoacán & Pseudothelphusa jouyi & Cambarellus montezumae & CNCR \\
\hline 16 & Jacona & Michoacán & Pseudothelphusa jouyi & Procambarus digueti & CNCR \\
\hline 17 & Tangancícuaro & Michoacán & Pseudothelphusa jouyi & $\begin{array}{l}\text { Cambarellus montezumae, } \\
\text { Procambarus digueti }\end{array}$ & CNCR \\
\hline 18 & Uruapan & Michoacán & Pseudothelphusa sp. & Procambarus bouvieri & CNCR \\
\hline 19 & Entre Cuautla y Yautepec & Morelos & Pseudothelphusa morelosis & Cambarellus montezumae & CNCR \\
\hline 20 & Las Estacas & Morelos & Pseudothelphusa morelosis & Cherax quadricarinatus & $\begin{array}{l}\text { CNCR, } \\
\text { Bortolini et al. } \\
\quad(2007)\end{array}$ \\
\hline 21 & Chignahuapan & Puebla & Pseudothelphusa americana & Procambarus contrerasi & CNCR \\
\hline 22 & Zacatlán & Puebla & Pseudothelphusa americana & Procambarus riojai & CNCR \\
\hline 23 & Tequisquiapan & Querétaro & Pseudothelphusa jouyi & Cambarellus montezumae & CNCR \\
\hline 24 & Mazatlán & Sinaloa & Pseudothelphusa sp. & Cambarellus occidentalis & CNCR \\
\hline 25 & Río Carrizal & Tabasco & Potamocarcinus hartmanni & $\begin{array}{c}\text { Procambarus llamasi, } P . \\
\text { acanthophorus }\end{array}$ & $\begin{array}{l}\text { CNCR, Álvarez } \\
\text { et al. (2005) }\end{array}$ \\
\hline 26 & Río González & Tabasco & Potamocarcinus hartmanni & Procambarus llamasi & $\begin{array}{l}\text { CNCR, Álvarez } \\
\text { et al. (2005) }\end{array}$ \\
\hline 27 & Teapa & Tabasco & $\begin{array}{l}\text { Potamocarcinus hartmanni, } \\
\text { Odontothelphusa monodontis }\end{array}$ & Procambarus llamasi & $\begin{array}{l}\text { CNCR, Álvarez } \\
\text { et al. (2005) }\end{array}$ \\
\hline 28 & El Carmen & Tlaxcala & Pseudothelphusa americana & Cambarellus montezumae & CNCR \\
\hline 29 & Amatlán de los Reyes & Veracruz & Tehuana complanata & Procambarus rodriguezi & CNCR \\
\hline 30 & Catemaco & Veracruz & Tehuana diabolis & $\begin{array}{l}\text { Procambarus vazquezae, } \\
\text { P. catemacoensis }\end{array}$ & $\begin{array}{l}\text { CNCR, Rojas et } \\
\text { al. (2000) }\end{array}$ \\
\hline 31 & Zapoapan de Cabañas & Veracruz & Tehuana veracruzana & Procambarus zapoapensis & CNCR \\
\hline 32 & San Andrés Tuxtla & Veracruz & Tehuana poglayenorum & Procambarus zapoapensis & CNCR \\
\hline
\end{tabular}


La zona de contacto entre las distribuciones de acociles y cangrejos ocurre principalmente a lo largo del eje neovolcánico desde los límites entre Puebla y Veracruz hasta Nayarit (Fig. 2). En específico, desde la zona de los lagos cráter del este de Puebla hacia el oeste, la especie de acocil más común que aparece simultáneamente con especies de cangrejos es Cambarellus (Cambarellus) montezumae (De Saussure, 1857), seguido de Procambarus (Mexicambarus) bouvieri (Ortmann, 1909) y en Jalisco y Michoacán, P. (Procambarus) digueti (Bouvier, 1897). En el lago de Chapala se encuentran adicionalmente Cambarellus (Cambarellus) chapalanus (Faxon, 1898), C. (C.) prolixus (Villalobos y Hobbs, 1981) y C. (C.) montezumae que también deben de tener contacto con los cangrejos (Cuadro 1). Por parte de los cangrejos, son Pseudothelphusa americana De Saussure, 1857 y P. jouyi Rathbun, 1893, las especies que más veces entran en contacto con acociles, principalmente en Jalisco y Michoacán. En Morelos, Pseudothelphusa morelosis Pretzmann, 1968, y C. (C.) montezumae son las especies que suelen aparecer juntas en una misma localidad; además, la recientemente introducida Cherax quadricarinatus se mantiene en contacto con Pseudothelphusa morelosis (Bortolini et al., 2007).

Hacia el sureste, en la región de Los Tuxtlas, se presenta una zona de contacto entre los 2 grupos en la que interviene otro de especies distintas (Fig. 2). Por parte de los acociles, Procambarus (Austrocambarus) zapoapensis Villalobos, 1954, P. (A.) vazquezae Villalobos, 1954 y $P$. (A.) catemacoensis Rojas, Álvarez y Villalobos, 2000 son las especies que están en contacto con Tehuana veracruzana Rodríguez y Smalley, 1969, T. poglayenorum (Pretzmann, 1980) y T. diabolis (Pretzmann, 1980) (Cuadro 1).

En Chiapas, el acocil Procambarus (Austrocambarus) mirandai Villalobos, 1954, es el que con mayor frecuencia está en contacto con cangrejos, especialmente con Raddaus bocourti (A. Milne Edwards, 1866); se han registrado áreas en Berriozábal, Bochil, Rayón y Montebello donde se distribuyen ambos (Fig. 3). En el municipio de Ocosingo, específicamente en arroyos cercanos a las ruinas de Toniná, existe otra zona de sobrelapamiento (Villalobos et al., 1989), no sólo entre cambáridos (Procambarus (Austrocambarus) sp.) y pseudotelfúsidos (Odontothelphusa toninae Álvarez y Villalobos, 1991), sino también con camarones palemónidos de la especie Cryphiops (Bithynops) villalobosi Villalobos, Nates y Cantú, 1989.

Otra manera de explorar las zonas de contacto de las 2 distribuciones es mediante el examen de la distribución altitudinal. Al respecto, es notorio que los acociles tienden en general a ocupar áreas más elevadas que los cangrejos. Entre los $19^{\circ}$ y los $21^{\circ}$ de latitud norte, que corresponde a la latitud del eje neovolcánico, se observa que los acociles

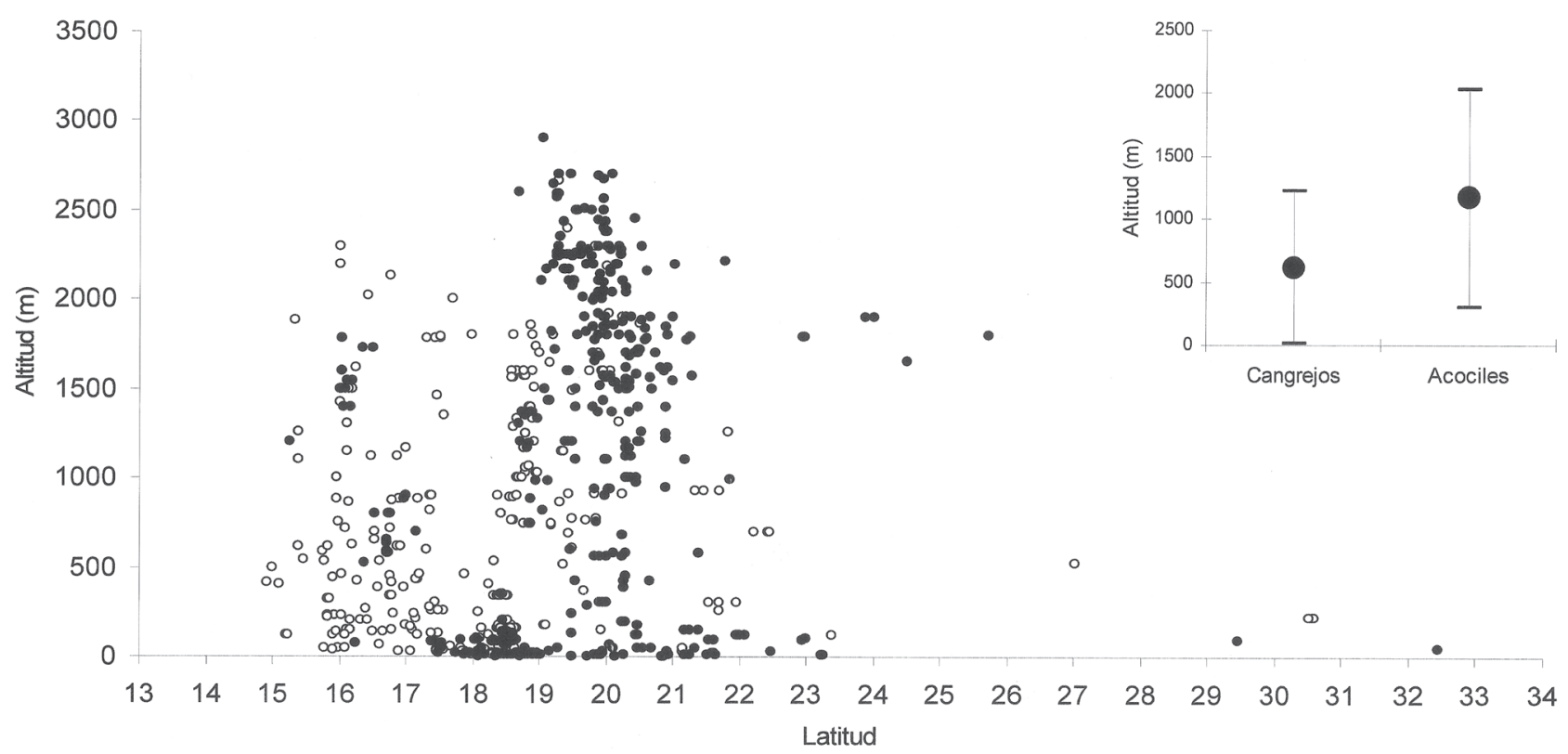

Figura 4. A, gráfica de la altitud versus latitud de las localidades que se analizan de cangrejos de agua dulce de la familia Pseudothelphusidae (círculos blancos) y de acociles de la familia Cambaridae (círculos negros); B, promedio \pm una desviación estándar de las altitudes de las localidades para cangrejos y acociles. 
tienden a distribuirse en localidades más altas que los cangrejos (Fig. 4A). De manera que es posible que la localidad de un acocil se encuentre muy cerca de la de un cangrejo, pero altitudinalmente estén muy separados. Con los datos considerados, el promedio de altitud de las localidades de uno y otro grupo son diferentes: $618 \pm 604 \mathrm{~m}$ para los cangrejos y $1169 \pm 867 \mathrm{~m}$ para los acociles (Fig. 4B).

\section{Discusión}

Los resultados muestran que las distribuciones de acociles y cangrejos se sobrelapan en regiones con condiciones distintas y con una variedad de especies y géneros. Es decir, que el sobrelapamiento entre ambos grupos no es específico de una región o de una o algunas especies en particular, sino que se da bajo una gran variedad de circunstancias. La falta de un patrón común con el que se puedan describir tanto los puntos de contacto como aquellas regiones donde éste no se presenta sugiere la idea de que ambas distribuciones han sido moldeadas independientemente. La siguiente es una argumentación de por qué se propone que la hipótesis de Rodríguez (1986) no explica la distribución de éstos 2 grupos en territorio mexicano.

En primer lugar, es notable el número y la diversidad de especies que están involucradas en las áreas de sobrelapamiento de las distribuciones; 17 de 60 especies (28\%) en los cangrejos, y 18 de 56 (32\%) en los acociles. Alrededor de un tercio de las especies en cada grupo están en los mismos sitios, lo que es una proporción muy alta para ser una interacción excluyente que se ha dado por largos periodos de tiempo.

Hobbs (1984) planteó que el centro de diversificación de los acociles del género Procambarus se encuentra en el sureste de los EUA y que desde ahí se presenta un trazo que, hacia el sur, bordea el golfo de México, llega a Guatemala y en un giro hacia el norte, alcanza Cuba. Esta hipótesis acepta una migración hacia el sur del stock ancestral. En cuanto al género Cambarellus, las especies del subgénero Cambarellus que se distribuyen en México pudieron haberse separado de las especies del sur de EUA hace unos 45 millones de años con 2 ramas principales, una en el centro-oriente y otra en el norte occidente de México (Pedraza, 2011).

Los cangrejos de la familia Pseudothelphusidae pudieron diversificarse a partir de un stock ancestral que quedó dividido, con una parte en Sudamérica y otra en Norteamérica, al separarse estas 2 masas de tierra al final del Cretácico. Un centro de diversificación se ha ubicado en el istmo de Tehuantepec y de ahí han radiado varios linajes hacia Centroamérica y hacia el norte de México para alcanzar su conformación actual (Villalobos y Álvarez, 2010a).
En ninguno de los 2 casos se ha recurrido a la exclusión de acociles por cangrejos para explicar sus patrones actuales de distribución. En cada grupo se han utilizado, principalmente, eventos geológicos para explicar la colonización de nuevas áreas y la posterior especiación. En ambos casos, la posibilidad de colonizar nuevas regiones ha estado asociada a la existencia de los hábitats particulares que cada grupo requiere; por ejemplo, la existencia de lagos para los acociles o de pequeños arroyos en las cabeceras de ríos para cangrejos, aunque esta diferencia pueda ser relativa.

La composición de las especies involucradas también arroja información pertinente. En cuanto a los cangrejos, hay 17 especies que pertenecen a 6 géneros distintos en 2 tribus: Raddaus, Phrygiopilus, Odontothelphusa y Potamocarcinus en Potamocarcinini; Pseudothelphusa y Tehuana en Pseudothelphusini. Aceptando, como lo proponen Villalobos y Álvarez (2010b), que los cangrejos pseudotelfúsidos se movieron del sur, desde Chiapas, hacia el norte, hasta Sonora, dentro del territorio mexicano y tomando en cuenta que los 6 géneros representan linajes muy distintos, tenemos que las especies registradas coexisitendo con acociles pueden tener edades muy diferentes. Esto sugiere que los tiempos en los cuales han entrado en contacto con acociles también pueden ser muy diferentes. Es probable que Pseudothelphusa americana, $P$. terrestris Rathbun, 1893, P. jouyi y P. seiferti Hobbs, 1980 sean especies muy antiguas, puesto que se encuentran al norte del eje neovolcánico que se formó durante el Oligoceno y Mioceno, posición que debieron alcanzar antes de la orogenia de esta cadena montañosa (Villalobos, 2005). En contraste, en la región de Los Tuxtlas, Veracruz, donde entran en contacto con acociles 3 especies de cangrejos del género Tehuana (T. diabolis, T. poglayenorum, T. veracruzana), el vulcanismo más intenso que moldeó la región empezó hace unos 7 millones de años y ha sido continuo hasta nuestros días (Martín-del Pozzo, 1997). En el extremo más reciente, Potamocarcinus hartmanni Pretzmann, 1975, tiene una distribución que se sobrelapa con la de 2 especies de acociles (Procambarus (Austrocambarus) acanthophorus Villalobos, 1948, y P. (A.) llamasi Villalobos, 1954) en la llanura costera, o planicie fluviodeltaica, de Tabasco que se desarrolla desde el Cuaternario $(<10000$ años; Ortiz-Pérez et al., 2005). Debido a la amplia variación en la edad de los linajes y en los posibles tiempos de contacto entre cangrejos y acociles resultaría aventurado proponer un escenario de competencia aplicable en todos los casos.

Con respecto a los acociles, igual que con los cangrejos, hay diversos linajes involucrados en las zonas de contacto. Además de las especies de Procambarus y Cambarellus, los 2 géneros nativos en México, está el parastácido Cherax 
quadricarinatus, nativo de Australia y recientemente introducido, interactuando con Pseudothelphusa morelosis en Las Estacas, Morelos, sin evidencia a lo largo de 5 años de que uno esté excluyendo al otro (Bortolini et al., 2007). Este ejemplo resulta de especial interés pues ilustra que $C$. quadricarinatus pudo establecerse en cuerpos de agua donde existían cangrejos, cuando la predicción derivada de la hipótesis de Rodríguez (1986) sería que eso no puede ocurrir.

Uno de los puntos de contacto más interesantes es el que se da en la zona de Uruapan y Tangancícuaro, Michoacán, donde se encuentran Procambarus (P.) digueti y Procambarus (M.) bouvieri, que están consideradas las especies más antiguas o más cercanas a los ancestros de los cambáridos que se presume aparecieron en esa región durante el Cretácico superior o medio (Villalobos, 1983; Hobbs, 1984). Ambas especies tienen una distribución desconectada del resto de las especies de Procambarus que se concentran a lo largo de la cuenca del golfo de México. Aunado a la presencia de estas 2 especies de Procambarus, en la región hay una importante diversidad del género Cambarellus. En particular, en la zona se tiene a $C$. $(C$.) montezumae, pero a tan sólo unas decenas de kilómetros está $C$. (C.) patzcuarensis Villalobos, 1943 en el lago de Pátzcuaro. La diversificación de ambos géneros ha ocurrido de manera independiente y en tiempos distintos; Breinholt et al. (2009) obtienen un tiempo de separación entre la línea de Cambarellus con el resto de los géneros de Cambaridae de 90 millones de años. Por su parte Pedraza (2011) obtiene que las especies de Cambarellus del centro de México se hayan originado entre los últimos 1.5 a 9 millones de años. Además, se presentan cangrejos de agua dulce Pseudothelphusa sp., en una zona aledaña, pero de menor altitud, en arroyos que vierten hacia la cuenca del Balsas. El punto importante es que las especies que ahora coexisten en la zona de Uruapan tienen orígenes muy diferentes y llegan a esta área por procesos independientes y en tiempos distintos que no están relacionados con la competencia.

En Chiapas, en las lagunas de Montebello coexisten en los mismos cuerpos de agua el acocil $P$. (A.) mirandai, que podría suponerse una de las formas más derivadas dentro del subgénero Austrocambarus, con cangrejos de linajes muy distintos, Raddaus bocourti y Phrygiopilus montebelloensis Álvarez y Villalobos, 1998. Se puede especular que aquí contrasta la presencia de un acocil muy derivado con la de 2 cangrejos que, por estar en un área considerada centro de diversidad para la familia Pseudothelphusidae, pueden ser especies muy antiguas. Además de Montebello, Procambarus (A.) mirandai y Raddaus bocourti coexisten en varios otros puntos dentro de Chiapas

Del análisis final se pueden derivar las siguientes conclusiones: 1) los patrones de distribución de cangrejos pseudotelfúsidos y de acociles tienen un sobrelapamiento importante a través de un amplio intervalo geográfico; 2) especies tanto de acociles como de cangrejos, pertenecientes a diferentes linajes y con orígenes también distintos, convergen en decenas de localidades distintas sin mostrar ningún patrón; 3) en las zonas de sobrelapamiento no se han documentado ni interacciones ni exclusiones de un grupo o de otro; 4) tanto acociles como cangrejos se siguen encontrando en muchas de las localidades donde están en contacto sin evidencia de que uno esté excluyendo al otro; 5) el microhábitat de cada grupo es diferente, por lo que hasta ahora no se ha registrado competencia directa entre cangrejos y acociles en la naturaleza y 6) el grupo de los cangrejos con afinidad tropical y el de los acociles con origen en regiones templadas han alcanzado sus distribuciones actuales de manera independiente y entran en contacto a lo largo de una gran zona de transición que corresponde aproximadamente a la frontera entre las regiones neártica y neotropical.

\section{Agradecimientos}

El primer autor agradece el apoyo otorgado mediante el proyecto PAPIIT IN214910-3 “Sistemática, distribución y conservación de los acociles (Crustacea: Cambaridae) de México".

\section{Literatura citada}

Álvarez, F. 1989. Smalleyus tricristatum, new genus, new species, and Pseudothelphusa parabelliana, new species (Brachyura, Pseudothelphusidae) from Los Tuxtlas, Veracruz, Mexico. Proceedings of the Biological Society of Washington 102:4549.

Álvarez, F. y J. L. Villalobos. 1991. Two new freshwater crabs from Mexico, Odontothelphusa toninae and Stygothelphusa lopezformenti (Crustacea, Brachyura, Pseudothelphusidae). Proceedings of the Biological Society of Washington 104:288-294.

Álvarez, F., J. L. Villalobos y R. Robles. 2005. Crustáceos. Capítulo 8. In Biodiversidad del estado de Tabasco, J. Bueno, F. Álvarez y S. Santiago (eds.). Instituto de Biología, UNAM/ CONABIO, México, D. F. p. 177-194.

Álvarez, F., J. L. Villalobos, M. Elías y G. Rivera. 2011. Crustáceos dulceacuícolas y terrestres de Chiapas. In Chiapas: estudios sobre su diversidad biológica, F. Álvarez (ed.). Universidad Nacional Autónoma de México, D. F. p. 209-297.

Álvarez, F., J. L. Villalobos y C. Hernández. En prensa. Perspectivas de estudios sobre los crustáceos de la región de Los Tuxtlas. In Avances y perspectivas en la investigación de bosques tropicales y sus alrededores: Los Tuxtlas, V. H. Reynoso y R. Coates (eds.). Instituto de Biología, UNAM, México, D. F. 
Armendáriz, G. 2011. Patrones de distribución y riqueza de especies de los acociles (Decapoda: Cambaridae) de México. Tesis, Maestría Posgrado en Ciencias Biológicas, Universidad Nacional Autónoma de México, México, D. F. 78 p.

Barbaresi, S. y F. Gherardi. 1997. Italian freshwater decapods: exclusion between the crayfish Austropotamobius pallipes (Faxon) and the crab Potamon fluviatile (Herbst). Bulletin française de la Pêche et de la Piscicolture 347:731-747.

Bortolini, J. L., F. Álvarez y G. Rodríguez-Almaraz. 2007. On the presence of the Australian redclaw crayfish, Cherax quadricarinatus, in Mexico. Biological Invasions 9:615620.

Botello, A. y F. Álvarez. 2010. Genetic variation in the stygobitic shrimp Creaseria morleyi (Decapoda: Palaemonidae), evidence of bottlenecks and re-invasions in the Yucatan Peninsula. Biological Journal of the Linnean Society 99:315325.

Breinholt, J., M. Pérez-Losada y K. A. Crandall. 2009. The timing of the diversification of the freshwater crayfishes. In Decapod crustacean phylogenetics, J. W. Martin, K. A. Crandall y D. L. Felder (eds.). CRC Press, Taylor \& Francis, New York. p. 343-356.

Butler, D. R. 2002. The environmental impact of crayfish bioturbation on a floodplain: Roanoke River, North Carolina Coastal Plain, U.S.A. Landform Analysis 3:35-40.

Campos, E. y G. Rodríguez-Almaraz. 1992. Distribution of the red swamp crayfish Procambarus clarkii (Girard, 1852) (Decapoda: Cambaridae) in Mexico: an update. Journal of Crustacean Biology 12:627-630.

Correia, A. M. y O. Ferreira. 1995. Burrowing behavior of the introduced red swamp crayfish Procambarus clarkii (Decapoda: Cambaridae) in Portugal. Journal of Crustacean Biology 15:248-257.

Covich, A. P. 2010. Winning the biodiversity arms race among freshwater gastropods: competition and coexistence through shell variability and predator avoidance. Hydrobiologia 653:191-215.

Cumberlidge, N. 1991. The respiratory system of Globonautes macropus (Rathbun, 1898), a terrestrial freshwater crab from Liberia (Gecarcinucoidea, Gecarcinucidae). Crustaceana 61:69-80.

Díaz, H. y G. Rodríguez. 1977. The branchial chamber of some terrestrial and semiterrestrial crabs. Biological Bulletin 153:485-506.

Gelder, S. R. 1999. Zoogeography of branchiobdellidans (Annelida) and temnocephalidans (Platyhelminthes) ectosymbiotic on freshwater crustaceans, and their reactions to one another in vitro. Hydrobiologia 406:21-31.

Gherardi, F. y A. Cioni. 2004. Agonism and interference competition in freshwater decapods. Behaviour 141:12971324.

Gherardi, F. y W. H. Daniels. 2004. Agonism and shelter competition between invasive and indigenous crayfish species. Canadian Journal of Zoology 82:1923-1932.

Harvey, B. C., J. L. White y R. J. Nakamoto. 2004. An emergent multiple predator effect may enhance biotic resistance in a stream fish assemblage. Ecology 85:127-133.

Hill, A. M. y D. M. Lodge. 1999. Replacement of resident crayfishes by an exotic crayfish: the roles of competition and predation. Ecological Applications 9:678-690.

Hobbs, H. H., Jr. 1981. The crayfishes of Georgia. Smithsonian Contributions to Zoology 318:1-549.

Hobbs, H. H., Jr. 1984. On the distribution of the crayfish genus Procambarus (Decapoda: Cambaridae). Journal of Crustacean Biology 4:12-24.

Hobbs, H. H., Jr. 1989. An illustrated checklist of the American crayfishes (Decapoda: Astacidae, Cambaridae and Parastacidae). Smithsonian Contributions to Zoology 480:1236.

Hobbs, H. H., III. 2001. Decapoda. In Ecology and classification of North American freshwater invertebrates, segunda edición, J. H. Thorp y A. P. Covich (eds.). Academic Press. p. 955-1001.

Holdich, D. y J. Black. 2007. The spiny-cheek crayfish, Orconectes limosus (Rafinesque, 1817) [Crustacea: Decapoda: Cambaridae], digs into the UK. Aquatic Invasions 2:1-15.

Holt, P. C. 1964. A new branchiobdellid (Annelida) from Costa Rica. Tulane Studies in Zoology 12:1-4.

Huidobro, L., J. J. Morrone, J. L. Villalobos y F. Alvarez. 2006. Distributional patterns of freshwater taxa (fishes, crustaceans and plants) from the Mexican Transition Zone. Journal of Biogeography 33:731-741.

Levine, J. M., P. B. Adler y S. G. Yelenik. 2004. A meta-analysis of biotic resistance to exotic plant invasions. Ecology Letters 7:975-989.

Martín-del Pozzo, A. L. 1997. Geología. In Historia natural de Los Tuxtlas, E. González-Soriano, R. Dirzo y R. C. Vogt (eds.). Instituto de Biología, UNAM/ CONABIO, México, D. F. p. 25-32.

Nakata, K. y S. Goshima. 2003. Competition for shelter or preferred sizes between the native crayfish species Cambaroides japonicus and the alien crayfish species Pacifastacus leniusculus in Japan in relation to prior residence, sex difference, and body size. Journal of Crustacean Biology 23:897-907.

Ortiz-Pérez, M. A., C. Siebe y S. Cram. 2005. Diferenciación ecogeográfica de Tabasco. In Biodiversidad del estado de Tabasco, J. Bueno, F. Álvarez y S. Santiago (eds.). Instituto de Biología, UNAM/ CONABIO, México, D. F. p. 305-322.

Pedraza, C. 2011. Patrones de diversificación y procesos evolutivos en cangrejos de río (Crustacea, Astacoidea). Tesis, Doctorado Universidad Complutense de Madrid, Madrid. $297 \mathrm{p}$.

Pretzmann, G. 1987. A contribution to a historic analysis of 
Mediterranean freshwater decapods chorology. Investigación Pesquera 51:17-25.

Rodríguez, G. 1982. Les crabes d'eau douce d'Amerique. Famille des Pseudothelphusidae. Faune Tropicale 22:1-223.

Rodríguez, G. 1986. Centers of radiation of freshwater crabs in the Neotropics. In Biogeography of the Crustacea, Crustacean, Issues 3, R. H. Gore y K. L. Heck (eds.). A. A. Balkema, Brookfield. Rotterdam. p. 51-67.

Rodríguez-Almaraz, G. y R. Muñiz-Martínez. 2008. Conocimiento de los acociles y langostinos del noreste de México: amenazas y propuestas de conservación. In Crustáceos de México: estado actual de su conocimiento, F. Álvarez y G. Rodríguez-Almaraz (eds.). Universidad Autónoma de Nuevo León/ PROMEP-SEP, México, D. F. p. 239-299.

Taylor, H. H. y P. Greenaway. 1979. The structure of the gills and lungs of the arid-zone crab, Holthuisana (Austrothelphusa) transversa (Martens) (Sundathelphusidae: Brachyura) including observations on arterial vessels within the gills. Journal of Zoology 189:359-384.

Torres, E. y F. Álvarez. 2012. Genetic variation in native and introduced populations of the red swamp crayfish Procambarus clarkii (Girard, 1852) (Crustacea, Decapoda, Cambaridae) in Mexico and Cost Rica. Aquatic Invasions 7:235-241.

Villalobos, A. 1982. Decapoda. In Aquatic biota of Mexico, Central America and the West Indies, S. H. Hulbert y A. Villalobos (eds.). San Diego State University, San Diego. p. 215-239.
Villalobos, A. 1983. The crayfishes of Mexico. Amerind, New Delhi. 276 p.

Villalobos, J. L. 2005. Sistemática de los cangrejos de agua dulce de México, Tribu Pseudothelphusini Ortmann, 1897 (Crustacea: Decapoda: Brachyura: Pseudothelphusidae). Análisis filogenético, biogeográfico y descripción de especies nuevas. Tesis doctoral, Facultad de Ciencias, Universidad Nacional Autónoma de México, México, D. F. 394 p.

Villalobos, J. L. y F. Álvarez. 2008. Los cangrejos de agua dulce de la familia Pseudothelphusidae (Decapoda: Brachyura: Eubrachyura) de México, con un apéndice de las especies citadas para América hasta 2006. In Crustáceos de México: estado actual de su conocimiento, F. Álvarez y G. RodríguezAlmaraz (eds.). Universidad Autónoma de Nuevo León/ PROMEP-SEP, México, D. F. p. 239-299.

Villalobos, J. L. y F. Álvarez. 2010a. A morphological phylogeny of the family Pseudothelphusidae. 21st Senckenberg Symposium on the Biology of freshwater decapods, 8-10 diciembre de 2010, Frankfurt.

Villalobos, J. L. y F. Álvarez. 2010b. Phylogenetic analysis of the Mexican freshwater crabs of the tribe Pseudothelphusini (Decapoda: Brachyura: Pseudothelphusidae). Zoological Journal of the Linnean Society 160:457-484.

Villalobos, J. L., J. C. Nates y A. Cantú. 1989. Revisión de los géneros Cryphiops Dana, 1852 y Bithynops Holthuis, 1973 de la familia Palaemonidae (Crustacea, Decapoda), con la descripción de una especie nueva para el estado de Chiapas, México. Anales del Instituto de Biología, Serie Zoología, 60:159-184. 